\title{
ON SQUARE-PRESERVING ISOMETRIES OF CONVOLUTION ALGEBRAS
}

\author{
SADAHIRO SAEKI
}

\begin{abstract}
Let $S$ and $S^{\prime}$ be two semigroups, each contained in a locally compact group. Under certain conditions on $S$ and $S^{\prime}$, we shall characterize those isometric additive surjections $T: M(S) \rightarrow M\left(S^{\prime}\right)$ which preserve convolution squares. Our results generalize the classical results of Wendel and of Johnson and also Patterson's characterization of isometric involutions on measure algebras.
\end{abstract}

Let $G$ be a locally compact group, and let $M(G)$ be the convolution measure algebra consisting of all regular complex Borel measures on $G$ (J. L. Taylor [13]). For each subsemigroup $S$ of $G$, let $M(S)$ be the set of all $\mu \in M(G)$ that are concentrated on $S$. Then $M(S)$ forms a norm-closed subalgebra of $M(G)$. Under certain conditions on two semigroups $S$ and $S^{\prime}$, each contained in a locally compact group, we shall characterize those isometric additive surjections $T: M(S) \rightarrow M\left(S^{\prime}\right)$ which preserve convolution squares: $T(\mu * \mu)=T \mu * T \mu$. Such a $T$ is either an isomorphism or an anti-isomorphism (as a mapping between two rings), and also either linear or conjugately linear (Theorem 7). Our characterization of such mappings generalizes not only the classical results of J. G. Wendel [14] and of B. E. Johnson [4] about isometric isomorphisms on measure algebras, but also P. L. Patterson's characterization of isometric involutions on measure algebras [7]. Our main result (Theorem 4) appears to be new even for finite groups.

Recall that a semigroup $S$ is said to satisfy the cancellation law if $x \neq y$ in $S$ implies $a x \neq a y$ and $x a \neq y a$ for each $a \in S$. Let $f: S \rightarrow S^{\prime}$ be a mapping from a semigroup (or a ring) into another. We say that $f$ is a semihomomorphism if it is either a homomorphism or an anti-homomorphism $(f(x y)=f(y) f(x))$. If, in addition, $f$ is a bijection, we call $f$ a semiisomorphism.

We begin with a purely algebraic result, which will play central role in our work.

Proposition 1. Let $S$ be a semigroup, let $S^{\prime}$ be a semigroup satisfying the cancellation law, and let $x \rightarrow x^{\prime}: S \rightarrow S^{\prime}$ be a mapping such that for each $x$ and $y \in S$, either $(x y)^{\prime}=x^{\prime} y^{\prime}$ or $(x y)^{\prime}=y^{\prime} x^{\prime}$. Then $x \rightarrow x^{\prime}$ is a semihomomorphism.

Received by the editors November 9, 1993 and, in revised form, May 30, 1994.

1991 Mathematics Subject Classification. Primary 43A10; Secondary 20M15.

Key words and phrases. Measure algebras, semihomomorphisms, Jordan products, tight convergence. 
Proof. We shall prove the result in five steps.

(I) $(x y)^{\prime}=x^{\prime} y^{\prime}$ if and only if $(y x)^{\prime}=y^{\prime} x^{\prime}$. Similarly, $(x y)^{\prime}=y^{\prime} x^{\prime}$ if and only if $(y x)^{\prime}=x^{\prime} y^{\prime}$.

To prove these, suppose $(x y)^{\prime}=x^{\prime} y^{\prime}$. Then $\left(x y^{2}\right)^{\prime}=x^{\prime}\left(y^{\prime}\right)^{2}$. For, otherwise, $\left(x y^{2}\right)^{\prime}=\left(y^{\prime}\right)^{2} x^{\prime}$. Hence

$$
\left(y^{\prime}\right)^{2} x^{\prime}=(x y \cdot y)^{\prime}=\left\{\begin{array}{l}
(x y)^{\prime} \cdot y^{\prime}=x^{\prime} y^{\prime} \cdot y^{\prime}, \quad \text { or } \\
y^{\prime} \cdot(x y)^{\prime}=y^{\prime} \cdot x^{\prime} y^{\prime} .
\end{array}\right.
$$

Since $S^{\prime}$ satisfies the cancellation law, it follows that $\left(y^{\prime}\right)^{2} x^{\prime}=x^{\prime}\left(y^{\prime}\right)^{2}=\left(x y^{2}\right)^{\prime}$ in either case. Therefore $\left(x y^{2}\right)^{\prime}=x^{\prime}\left(y^{\prime}\right)^{2}$ whenever $(x y)^{\prime}=x^{\prime} y^{\prime}$.

Suppose to the contrary that $(x y)^{\prime}=x^{\prime} y^{\prime}$ but $(y x)^{\prime} \neq y^{\prime} x^{\prime}$. Then $(y x)^{\prime}=$ $x^{\prime} y^{\prime}$, and so

$$
\begin{aligned}
x^{\prime} y^{\prime} \cdot x^{\prime} y^{\prime} & =(x y \cdot y x)^{\prime}=\left(x y^{2} \cdot x\right)^{\prime} \\
& =\left\{\begin{array}{l}
\left(x y^{2}\right)^{\prime} \cdot x^{\prime}=x^{\prime}\left(y^{\prime}\right)^{2} x^{\prime}, \quad \text { or } \\
x^{\prime}\left(x y^{2}\right)^{\prime}=x^{\prime} \cdot x^{\prime}\left(y^{\prime}\right)^{2} .
\end{array}\right.
\end{aligned}
$$

Hence $x^{\prime}$ and $y^{\prime}$ commute in either case, which contradicts $x^{\prime} y^{\prime}=(y x)^{\prime} \neq$ $y^{\prime} x^{\prime}$. The second assertion follows from the first assertion.

(II) $(x y x)^{\prime}=x^{\prime} y^{\prime} x^{\prime}$ for $x, y \in S$.

First suppose $(x y)^{\prime}=x^{\prime} y^{\prime}$. Then $\left(x y^{2}\right)^{\prime}=x^{\prime}\left(y^{\prime}\right)^{2}=(x y)^{\prime} \cdot y^{\prime}$, as was shown in the above proof. Hence $(y \cdot x y)^{\prime}=y^{\prime} \cdot(x y)^{\prime}=y^{\prime} x^{\prime} y^{\prime}$ by (I) with $(x, y)$ replaced by $(x y, y)$. By (I), we may exchange $x$ and $y$ in this conclusion to get $(x y x)^{\prime}=x^{\prime} y^{\prime} x^{\prime}$.

Next suppose $(x y)^{\prime}=y^{\prime} x^{\prime}$, but $(x y \cdot x)^{\prime} \neq x^{\prime}(x y)^{\prime}=x^{\prime} y^{\prime} x^{\prime}$. Then we must have $(x y \cdot x)^{\prime}=(x y)^{\prime} x^{\prime}$, which equals $y^{\prime}\left(x^{\prime}\right)^{2}$. Hence

$$
\begin{aligned}
y^{\prime} x^{\prime} \cdot y^{\prime} x^{\prime} & =(x y \cdot x y)^{\prime}=(x y x \cdot y)^{\prime} \\
& =\left\{\begin{array}{l}
(x y x)^{\prime} \cdot y^{\prime}=y^{\prime}\left(x^{\prime}\right)^{2} \cdot y^{\prime}, \text { or } \\
y^{\prime} \cdot(x y x)^{\prime}=y^{\prime} \cdot y^{\prime}\left(x^{\prime}\right)^{2} .
\end{array}\right.
\end{aligned}
$$

In either case, $x^{\prime}$ and $y^{\prime}$ commute, which yields a contradiction.

(III) Suppose $x, y, z \in S$,

$$
(x y)^{\prime}=x^{\prime} y^{\prime} \neq y^{\prime} x^{\prime} \text { and }(y z)^{\prime}=y^{\prime} z^{\prime} .
$$

Then we have $(x z)^{\prime}=x^{\prime} z^{\prime}$.

In fact, suppose to the contrary that $(x z)^{\prime} \neq x^{\prime} z^{\prime}$. Then

$$
(x z)^{\prime}=z^{\prime} x^{\prime} \neq x^{\prime} z^{\prime}=(z x)^{\prime},
$$

where the second equality follows from (I). Moreover,

$$
\begin{aligned}
z^{\prime} \cdot x^{\prime} y^{\prime} \cdot z^{\prime} & =(z \cdot x y \cdot z)^{\prime} \quad \text { by (II) and (1) } \\
& =(z x \cdot y z)^{\prime} \\
& =\left\{\begin{array}{l}
(z x)^{\prime}(y z)^{\prime}=x^{\prime} z^{\prime} \cdot y^{\prime} z^{\prime}, \text { or } \\
(y z)^{\prime}(z x)^{\prime}=y^{\prime} z^{\prime} \cdot x^{\prime} z^{\prime}
\end{array}\right.
\end{aligned}
$$

by (1) and (2). In the first case, $z^{\prime} x^{\prime}=x^{\prime} z^{\prime}$, which contradicts (2). Therefore we must have

$$
z^{\prime} x^{\prime} y^{\prime}=y^{\prime} z^{\prime} x^{\prime} \quad \text { and } \quad y^{\prime} z^{\prime} \neq z^{\prime} y^{\prime}
$$


(If $y^{\prime} z^{\prime}=z^{\prime} y^{\prime}$, then the equality in (3) would yield $x^{\prime} y^{\prime}=y^{\prime} x^{\prime}$, which contradicts (1).) Moreover, writing $z x y=z \cdot x y$ and applying (1), we get

$$
(z x y)^{\prime}=z^{\prime} x^{\prime} y^{\prime} \text { or } x^{\prime} y^{\prime} z^{\prime} .
$$

From $z x y=z x \cdot y$ and (2), we also get

$$
(z x y)^{\prime}=x^{\prime} z^{\prime} y^{\prime} \text { or } y^{\prime} x^{\prime} z^{\prime} \text {. }
$$

Suppose $(z x y)^{\prime}=z^{\prime} x^{\prime} y^{\prime}$. Then $z^{\prime} x^{\prime} y^{\prime}=y^{\prime} x^{\prime} z^{\prime}$ by (5) and (2); hence $y^{\prime} z^{\prime} x^{\prime}=y^{\prime} x^{\prime} z^{\prime}$ by (3), so $z^{\prime} x^{\prime}=x^{\prime} z^{\prime}$, which contradicts (2). Therefore we must have $(z x y)^{\prime}=x^{\prime} y^{\prime} z^{\prime}$ by (4). Hence (5) shows that either $x^{\prime} y^{\prime} z^{\prime}=x^{\prime} z^{\prime} y^{\prime}$ or $x^{\prime} y^{\prime} z^{\prime}=y^{\prime} x^{\prime} z^{\prime}$. In either case, we get a contradiction by either (3) or (1). Therefore (1) implies $(x z)^{\prime}=x^{\prime} z^{\prime}$.

(IV) Let $x \in S$ be given. If there exists $y \in S$ such that

$$
(x y)^{\prime}=x^{\prime} y^{\prime} \neq y^{\prime} x^{\prime}
$$

then $(x z)^{\prime}=x^{\prime} z^{\prime}$ for each $z \in S$.

To prove this, fix any $y \in S$ satisfying (6) and any $z \in S$. Suppose to the contrary that $(x z)^{\prime} \neq x^{\prime} z^{\prime}$. Then

$$
(x z)^{\prime}=z^{\prime} x^{\prime} \neq x^{\prime} z^{\prime}=(z x)^{\prime} .
$$

Therefore $(y z)^{\prime}=y^{\prime} z^{\prime}$ cannot happen by (III). Hence

$$
(y z)^{\prime}=z^{\prime} y^{\prime} \neq y^{\prime} z^{\prime} \text {. }
$$

Note that

$$
(x y z)^{\prime}=(x y \cdot z)^{\prime}=x^{\prime} y^{\prime} z^{\prime} \text { or } z^{\prime} x^{\prime} y^{\prime}
$$

by (6), and

$$
(x y z)^{\prime}=(x \cdot y z)^{\prime}=x^{\prime} z^{\prime} y^{\prime} \text { or } z^{\prime} y^{\prime} x^{\prime}
$$

by (8). Therefore, in light of (7) and (8),

$$
(x y z)^{\prime}=x^{\prime} y^{\prime} z^{\prime}=z^{\prime} y^{\prime} x^{\prime}
$$

is the unique possibility. In particular, $(x y \cdot z)^{\prime}=(x y)^{\prime} z^{\prime}$ by (6), and so (I) ensures that

$$
(z x y)^{\prime}=z^{\prime}(x y)^{\prime}=z^{\prime} x^{\prime} y^{\prime} .
$$

Finally, consider the following three expansions of $(y z x y)^{\prime}$ :

$$
\begin{gathered}
(y \cdot z x \cdot y)^{\prime}=y^{\prime} x^{\prime} z^{\prime} y^{\prime} \quad \text { by (II) and (7), } \\
(y \cdot z x y)^{\prime}=y^{\prime} z^{\prime} x^{\prime} y^{\prime} \quad \text { or } \quad z^{\prime} x^{\prime} y^{\prime} y^{\prime} \quad \text { by (10), } \\
(y z \cdot x y)^{\prime}=z^{\prime} y^{\prime} x^{\prime} y^{\prime} \quad \text { or } \quad x^{\prime} y^{\prime} z^{\prime} y^{\prime} \quad \text { by (8) and (6). }
\end{gathered}
$$

Since $x^{\prime} z^{\prime} \neq z^{\prime} x^{\prime}$, we have $y^{\prime} x^{\prime} z^{\prime}=z^{\prime} x^{\prime} y^{\prime}$ by (11) and (12). Since $x^{\prime} y^{\prime} \neq y^{\prime} x^{\prime}$, we also have $y^{\prime} x^{\prime} z^{\prime}=z^{\prime} y^{\prime} x^{\prime}$ by (11) and (13). But then $x^{\prime} y^{\prime}=y^{\prime} x^{\prime}$, a contradiction.

(V) Set

$$
\begin{aligned}
& A=\left\{x \in S:(x z)^{\prime}=x^{\prime} z^{\prime} \forall z \in S\right\} \\
& B=\left\{x \in S:(x z)^{\prime}=z^{\prime} x^{\prime} \forall z \in S\right\}
\end{aligned}
$$


If $x \in S$ and $(x y)^{\prime}=x^{\prime} y^{\prime} \neq y^{\prime} x^{\prime}$ for some $y \in S$, then $x \in A$ by (IV). Hence $x \notin A$ implies that, for each $y \in S$, either $(x y)^{\prime}=y^{\prime} x^{\prime}$ or $x^{\prime} y^{\prime}=y^{\prime} x^{\prime}$, so $(x y)^{\prime}=y^{\prime} x^{\prime}$ in either case; hence $x \in B$. Thus we have proved that $S=A \cup B$.

Suppose to the contrary that neither $A \subset B$ nor $B \subset A$. Then there exist $x, y \in S$ such that $x \in A \backslash B$ and $y \in B \backslash A$. Note that $(x y)^{\prime}=x^{\prime} y^{\prime}$. Since $S=A \cup B$, we have either $x y \in A$ or $x y \in B$. First suppose that $x y \in A$. Then $z \in S$ implies

$$
\begin{aligned}
x^{\prime} y^{\prime} z^{\prime} & =(x y)^{\prime} z^{\prime}=(x y z)^{\prime} \\
& =x^{\prime}(y z)^{\prime} \quad \text { since } x \in A \\
& =x^{\prime} z^{\prime} y^{\prime} \quad \text { since } y \in B .
\end{aligned}
$$

Hence $(y z)^{\prime}=z^{\prime} y^{\prime}=y^{\prime} z^{\prime}$ for every $z \in S$; that is, $y \in A$, which contradicts our choice of $y$. Therefore we must have $x y \in B$. But then $z \in S$ implies

$$
\begin{aligned}
z^{\prime} x^{\prime} y^{\prime} & =z^{\prime}(x y)^{\prime}=(x y z)^{\prime} \quad \text { since } x y \in B \\
& =x^{\prime}(y z)^{\prime} \quad \text { since } x \in A \\
& =x^{\prime} z^{\prime} y^{\prime} \quad \text { since } y \in B .
\end{aligned}
$$

Hence $z^{\prime} x^{\prime}=x^{\prime} z^{\prime}$, so $(x z)^{\prime}=z^{\prime} x^{\prime}$ for every $z \in S$. By the definition of $B$, this means $x \in B$, which contradicts our choice of $x$.

Thus we have proved that either $A \subset B$ or $B \subset A$. Since $S=A \cup B$, we conclude that either $S=A$ or $S=B$, which is nothing but the desired conclusion.

Remarks. Proposition 1 (Hua's theorem) is proved in E. Artin's book [1, pp. 39-40] for additive mappings $x \rightarrow x^{\prime}: S \rightarrow S^{\prime}$ from a field into another. However, the proof there is not applicable to our case because the additivity of $x \rightarrow x^{\prime}$ plays an essential role in that proof. For generalizations of Hua's theorem in some other settings, see N. Jacobson and C. E. Rickart [3], W. R. Scott [10], and L. N. Ševrin [11] and [12].

The following result characterizes the mappings $\beta: S \rightarrow S^{\prime}$ of the form $\beta(x)=z h(x)(x \in S)$, where $h: S \rightarrow S^{\prime}$ is a semihomomorphism and $z \in S^{\prime}$ is an element which commutes with every element of $h(S)$.

Corollary 2. Suppose $S$ is a semigroup with an identity $e$, and $S^{\prime}$ is a semigroup satisfying the cancellation law. Then a mapping $\alpha: S \rightarrow S^{\prime}$ is a semihomomorphism if and only if there exists a mapping $\beta: S \rightarrow S^{\prime}$ such that

$$
\{\beta(x y), \beta(y x)\}=\{\beta(x) \alpha(y), \alpha(y) \beta(x)\} \forall x \text { and } y \in S .
$$

Moreover, if $\beta$ is a mapping satisfying (i), then $\beta(x)=\beta(e) \alpha(x)=\alpha(x) \beta(e)$ for each $x \in S$.

Proof. One direction is obvious. To prove the nontrivial direction, let $\alpha$ and $\beta: S \rightarrow S^{\prime}$ be two mappings satisfying (i). Taking $x=e$ in (i), we get $\beta(y)=$ $\beta(e) \alpha(y)=\alpha(y) \beta(e)$ for each $y \in S$. Replacing $y$ by $x y$ in the last representation of $\beta$, we infer from (i) that $\{\alpha(x y), \alpha(y x)\}=\{\alpha(x) \alpha(y), \alpha(y) \alpha(x)\}$ because $S^{\prime}$ satisfies the cancellation law. Hence $\alpha$ is a semihomomorphism by Proposition 1, which completes the proof.

Now let $G$ be a locally compact group, and let $M(G)$ be the convolution measure algebra on $G$ (cf. E. Hewitt-K. A. Ross [2] and Taylor [13]). Recall 
that an $L$-subspace of $M(G)$ is a closed subspace $X$ of $M(G)$ such that $\mu \in$ $M(G), \nu \in X$, and $\mu \ll|\nu|$ implies $\mu \in X$ [13]. For each $E \subset G$, let $E^{0}$ be the interior of $E$ and let $M(E)$ be the set of all $\mu \in M(G)$ that are concentrated on $\sigma$-compact subsets of $E$ (depending on $\mu$ ). Thus $M(E)$ is an $L$-subspace of $M(G)$, and if $E$ is a subsemigroup of $G$, then $M(E)$ is also a subalgebra of $M(G)$. Let $\lambda_{G}$ be a left Haar measure on $G$, and let

$$
M_{a}(E)=\left\{\mu \in M(E): \mu \ll \lambda_{G}\right\} .
$$

Thus $M_{a}(G)$ is a two-sided $L$-ideal in $M(G)$.

In the sequel, let $G$ and $G^{\prime}$ be two locally compact groups, and let $S$ and $S^{\prime}$ be (not necessarily closed) subsemigroups of $G$ and of $G^{\prime}$, respectively.

Definition 3. (a) A net $\left(\mu_{n}\right)$ in $M(G)$ is said to be tight if it is norm-bounded and if for each $\varepsilon>0$, there exists a compact set $K \subset G$ such that eventually $\left|\mu_{n}\right|(G \backslash K)<\varepsilon$ (M. Loève [6]).

(b) Let $X \subset M(G)$ and $\mu \in M(G)$. A tight net for $\mu$ in $X$ means a net $\left(\mu_{n}\right)$ in $X$ which is tight as a net in $M(G)$ and converges to $\mu$ in the weak-* topology of $M(G)$.

(c) Let $X \subset M(G)$. A mapping $A: X \rightarrow M\left(G^{\prime}\right)$ is said to be tightly continuous if whenever $\mu \in X$ and $\left(\mu_{n}\right)$ is a tight net for $\mu$ in $X$, then $A \mu_{n} \rightarrow A \mu$ in the weak-* topology of $M\left(G^{\prime}\right)$.

Let $C(G)$ be the space of all bounded continuous functions on $G$. It is a well-known fact that if $\mu \in M(G)$ and $\left(\mu_{n}\right)$ is a tight net for $\mu$ in $M(G)$, then $\mu_{n} \rightarrow \mu$ in the weak topology of $M(G)$ induced by $C(G)$ (cf. [6]).

In order to give a motivation for our next result, let $A: \mathscr{R} \rightarrow \mathscr{R}^{\prime}$ be an additive mapping from a ring into another which preserves squares. Then we may apply $A$ to both sides of $x y+y x=(x+y)^{2}-x^{2}-y^{2}$ to obtain

$$
A(x y+y x)=(A x) A y+(A y) A x \text {. }
$$

In other words, $A$ preserves Jordan products. Now fix any $z^{\prime} \in \mathscr{R}^{\prime}$ which commutes with every $A x(x \in \mathscr{R})$, and define $B x=z^{\prime}(A x)$ for $x \in \mathscr{R}$. Then (I) ensures that

$$
B(x y+y x)=(B x) A y+(A y) B x .
$$

Our next result shows that, under certain strong assumptions on $A$ and $B,\left(\mathrm{~J}^{\prime}\right)$ implies (I) and $B$ is obtained in the above fashion (and much more).

Theorem 4. Suppose (i) $S \subset G$ is a subsemigroup with $S^{0} \neq \varnothing$ and $e \in \bar{S}$, (ii) each of $A, B: M(S) \rightarrow M\left(G^{\prime}\right)$ is an additive isometry onto a weak-* closed $L$-subspace of $M\left(G^{\prime}\right)$, and (iii) $A$ and $B$ satisfy

$$
B(\mu * \nu+\nu * \mu)=B \mu * A \nu+A \nu * B \mu \forall \mu, \nu \in M(S) .
$$

Then $S$ is necessarily closed, and there exist a unique continuous homomorphism $\gamma: S \rightarrow \mathbb{T}$ and a unique continuous semi-isomorphism $\alpha$ from $S$ onto a closed subsemigroup of $G^{\prime}$ such that either

(a) $A \mu=(\gamma \mu) \circ \alpha^{-1} \forall \mu \in M(S)$, or

(b) $A \mu=(\gamma \bar{\mu}) \circ \alpha^{-1} \forall \mu \in M(S)$.

In particular, $A$ is a semihomomorphism which is either linear or conjugately linear. Moreover, there exists a unique $c \in \mathbb{T}$ and a unique $z \in G^{\prime}$ such that $B \mu=\left(c \delta_{z}\right) * A \mu=(A \mu) *\left(c \delta_{z}\right)$ for each $\mu \in M(S)$. 
To prove this result, we need three lemmas. The first one of them is well known and so we shall only give a sketchy proof to it.

Lemma 4.1. (i) For each $\mu \in M(G)$, the mappings $\nu \rightarrow \mu * \nu$ and $\nu \rightarrow \nu * \mu$ are weak-* continuous on $M(G)$.

(ii) If $\left(\mu_{n}\right)$ [resp. $\left.\left(\nu_{n}\right)\right]$ is a tight net for $\mu$ [resp. $\left.\nu\right]$ in $M(G)$, then $\left(\mu_{n} * \nu_{n}\right)$ is a tight net for $\mu * \nu$ in $M(G)$.

(iii) If $\left(\mu_{n}\right)$ is a tight net for $\mu$ in $M(G)$ and if $\nu \in M_{a}(G)$, then $\mu_{n} * \nu \rightarrow \mu * \nu$ and $\nu * \mu_{n} \rightarrow \nu * \mu$ both in norm.

Proof. (i) is obvious.

(ii) For $\mu \in M(G), f \in C(G)$, and $y \in G$, set $(\mu \cdot f)(y)=\int f(x y) d \mu(x)$. Then $\mu \cdot f \in C(G)$ and $|(\mu \cdot f)(y)| \leq\|\mu\|_{M}\|f\|_{u}$ for each $y \in G$. Now let $\left(\mu_{n}\right),\left(\nu_{n}\right), \mu$, and $\nu$ be as in (ii). Plainly $\left(\mu_{n} * \nu_{n}\right)$ is a tight net in $M(G)$. If $f \in C(G)$, then $\left(\mu_{n} \cdot f\right)$ is a uniformly bounded net in $C(G)$ and converges to $\mu \cdot f$ uniformly on each compact subset of $G$. Therefore $\int f d\left(\mu_{n} * \nu_{n}\right)=\int\left(\mu_{n} \cdot f\right) d \nu_{n} \rightarrow \int(\mu \cdot f) d \nu$.

(iii) Replacing each $\mu_{n}$ by $\mu_{n}-\mu$, we may suppose $\mu=0$. If $f \in C_{c}(G)$, then $\mu_{n} * f \rightarrow 0$ uniformly on $G$ and the measures $\mu_{n} *\left(f \lambda_{G}\right)=\left(\mu_{n} * f\right) \lambda_{G}$ form a tight net in $M(G)$ by (ii). Hence $\left\|\mu_{n} * f\right\|_{1} \rightarrow 0$. Since $C_{c}(G)$ is dense in $L_{1}(G)$, it follows that $\left\|\mu_{n} * \nu\right\| \rightarrow 0$ for each $\nu \in M_{a}(G)$. Similarly $\left\|\nu * \mu_{n}\right\| \rightarrow 0$ for each $\nu \in M_{a}(G)$, as desired.

Lemma 4.2. Let $\mu \in M(G)$ be given. If there exists $x \in G$ such that

$$
\mu * \delta_{y}+\delta_{y} * \mu=0
$$

for $y=x$ and $x^{2}$, then $\mu=0$.

Proof. Let $D$ be the set of all $y \in G$ that satisfy $(*)$. Then $D$ is a symmetric closed subset of $G$. Moreover, $y \in G$ belongs to $D$ if and only if $\delta_{y-1} * \mu * \delta_{y}=$ $-\mu$. Hence $D^{3}=D$. Accordingly the set $D^{2}$ is a subgroup of $G$ (unless $D=\varnothing)$ and $y D^{2}=D$ whenever $y \in D$. If both $x$ and $x^{2}$ belong to $D$, then $e \in D^{2}=x^{2} D^{2}=D$ and therefore $2 \mu=0$ by $(*)$ with $y=e$, as desired.

Lemma 4.3. Let $S \subset G$ be a subsemigroup with nonempty interior, and let $A, B, C, D: M(S) \rightarrow M\left(G^{\prime}\right)$ be (not necessarily additive) mappings such that

$$
A(\mu * \nu+\nu * \mu)=B \mu * C \nu+C \nu * D \mu \forall \mu, \nu \in M(S) \text {. }
$$

Suppose $A$ is a norm-continuous injection, $C$ is a bounded mapping, and $C(M(S))$ is weak-* closed in $M\left(G^{\prime}\right)$. Then $S$ is necessarily closed in $G$ and $C$ is tightly continuous.

Proof. Given $\nu \in M(\bar{S}), M(S)$ has a tight net $\left(\nu_{n}\right)$ for $\nu$. Since $C$ is a bounded mapping, $\left(C \nu_{n}\right)$ is a norm-bounded net in $M\left(G^{\prime}\right)$, so it has a weak* cluster point $\nu^{\prime} \in M\left(G^{\prime}\right)$. To prove $\nu \in M(S)$ and $C \nu=\nu^{\prime}$, fix any $\mu \in M_{a}(S)$. Then $\mu * \nu_{n}+\nu_{n} * \mu \rightarrow \mu * \nu+\nu * \mu$ in norm by Lemma 4.1(iii); in particular, $\mu * \nu+\nu * \mu \in M(S)$. It follows from the continuity of $A$ that

$$
\begin{aligned}
A(\mu * \nu+\nu * \mu) & =\lim A\left(\mu * \nu_{n}+\nu_{n} * \mu\right) \\
& =\lim \left\{B \mu * C \nu_{n}+C \nu_{n} * D \mu\right\} \quad \text { by }(*) \\
& =B \mu * \nu^{\prime}+\nu^{\prime} * D \mu,
\end{aligned}
$$

where the last equality follows from Lemma 4.1(i). 
Now $C(M(S))$ is weak-* closed in $M\left(G^{\prime}\right)$; hence there exists $\sigma \in M(S)$ such that $C \sigma=\nu^{\prime}$. Since $A$ is injective, it follows from (*) and (1) that

$$
\mu *(\nu-\sigma)+(\nu-\sigma) * \mu=0 \forall \mu \in M_{a}(S) .
$$

For each $x \in S^{0}, M_{a}(S)$ has a tight net for $\delta_{x}$. Therefore (2) combined with Lemma 4.1(i) ensures that $\delta_{x} *(\nu-\sigma)+(\nu-\sigma) * \delta_{x}=0$ for each $x \in S^{0}$. But $S^{0}$ is obviously a semigroup and $S^{0} \neq \varnothing$ by hypothesis. It follows from Lemma 4.2 that $\nu-\sigma=0$; hence $\nu=\sigma \in M(S)$ and $C \nu=\nu^{\prime}$. As $\nu^{\prime}$ was an arbitrary weak-* cluster point of the norm-bounded net $\left(C \nu_{n}\right)$ in $M\left(G^{\prime}\right)$, we conclude that $C \nu_{n} \rightarrow C \nu$ weak-* .

Thus we have confirmed that $M(\bar{S})=M(S)$ and $C$ is tightly continuous. In particular, $S$ is closed in $G$, which completes the proof.

Proof of Theorem 4. (I) Let $x \in S$ be given. Then there exists a unique $\gamma(x) \in \mathbb{T}$ and a unique $\alpha(x) \in G^{\prime}$ such that

$$
A\left(\delta_{x}\right)=\gamma(x) \delta_{\alpha(x)}
$$

Similarly, there exists a unique $\chi(x) \in \mathbb{T}$ and a unique $\beta(x) \in G^{\prime}$ such that

$$
B\left(\delta_{x}\right)=\chi(x) \delta_{\beta(x)} \text {. }
$$

To confirm these facts, note that two bounded measures $\mu$ and $\nu$ are mutually singular if and only if $\|\mu \pm \nu\|=\|\mu\|+\|\nu\|$ (S. Kakutani [5]). Since $A$ is an additive isometry, it follows that $A$ preserves the mutual singularity of measures. Moreover, $A(M(S))$ is an $L$-subspace of $M\left(G^{\prime}\right)$ by (ii). Therefore the support of $A\left(\delta_{x}\right)$ is a singleton and $\left\|A\left(\delta_{x}\right)\right\|=\left\|\delta_{x}\right\|=1$. Hence there exist a unique $\gamma(x) \in \mathbb{T}$ and a unique $\alpha(x) \in G^{\prime}$ satisfying (1). The proof for $B$ is the same.

(II) The function $\gamma: S \rightarrow \mathbb{T}$ is a homomorphism and $\alpha: S \rightarrow G^{\prime}$ is a semihomomorphism. Moreover, $\chi(x)=\chi(e) \gamma(x)$ and $\beta(x)=\beta(e) \alpha(x)=$ $\alpha(x) \beta(e)$ for each $x \in S$. (Later, we shall show $e \in S$.)

In fact, let $x, y \in S$ be given. Then

$$
\chi(x y) \delta_{\beta(x y)}+\chi(y x) \delta_{\beta(y x)}=B\left(\delta_{x y}+\delta_{y x}\right) \text { by (2) }
$$

$$
\begin{aligned}
& =\left(B \delta_{x}\right) *\left(A \delta_{y}\right)+\left(A \delta_{y}\right) *\left(B \delta_{x}\right) \quad \text { by }(\mathbf{J}) \\
& =\chi(x) \gamma(y)\left\{\delta_{\beta(x) \alpha(y)}+\delta_{\alpha(y) \beta(x)}\right\} \quad \text { by (1) and (2). }
\end{aligned}
$$

Hence $\chi(x y)=\chi(y x)=\chi(x) \gamma(y)$ and

$$
\{\beta(x y), \beta(y x)\}=\{\beta(x) \alpha(y), \alpha(y) \beta(x)\} .
$$

This, combined with Corollary 2, establishes the results.

(III) We have

$$
\begin{gathered}
A\left(i \delta_{x}\right)=i \gamma(x) \delta_{\alpha(x)} \forall x \in S, \text { or } \\
A\left(i \delta_{x}\right)=-i \gamma(x) \delta_{\alpha(x)} \forall x \in S .
\end{gathered}
$$

Moreover, $A$ satisfies (4.A) or (5.A) if and only if $B$ satisfies

$$
\begin{gathered}
B\left(i \delta_{x}\right)=i \chi(x) \delta_{\beta(x)} \forall x \in S, \text { or } \\
B\left(i \delta_{x}\right)=-i \chi(x) \delta_{\beta(x)} \forall x \in S,
\end{gathered}
$$

respectively. 
To see these, we first argue as in (I) to get $A\left(i \delta_{x}\right)=\gamma^{\prime}(x) \delta_{\alpha^{\prime}(x)}$ for some $\gamma^{\prime}(x) \in \mathbb{T}$ and some $\alpha^{\prime}(x) \in G^{\prime}$. By comparing the norms of both sides of $A\left(\delta_{x}+i \delta_{x}\right)=\gamma(x) \delta_{\alpha(x)}+\gamma^{\prime}(x) \delta_{\alpha^{\prime}(x)}$, we see that $\alpha(x)=\alpha^{\prime}(x)$ and $\gamma^{\prime}(x)=$ $\pm i \gamma(x)$. Hence, for each fixed $x \in S$, either (4.A) or (5.A) holds. Similarly, for each fixed $x \in S$, either (4.B) or (5.B) holds.

Suppose to the contrary that there exist $x, y \in S$ such that $x$ satisfies (4.B) but $y$ satisfies (5.A) with $x$ replaced by $y$. Then

$$
\begin{aligned}
\chi(x) \gamma(y)\left\{\delta_{\beta(x y)}+\delta_{\beta(x y)}\right\} & =B\left(\delta_{x y}+\delta_{y x}\right) \quad \text { by }(3) \\
& =-B\left(i \delta_{x} * i \delta_{y}+i \delta_{y} * i \delta_{x}\right) \\
& =-\left\{B\left(i \delta_{x}\right) * A\left(i \delta_{y}\right)+A\left(i \delta_{y}\right) * B\left(i \delta_{x}\right)\right\} \\
& =-\chi(x) \gamma(y)\left\{\delta_{\beta(x) \alpha(y)}+\delta_{\alpha(y) \beta(x)}\right\},
\end{aligned}
$$

which is of course absurd. Therefore, if (4.B) holds for some $x \in S$, then (4.A) holds for every $x \in S$. Similarly, if (4.A) holds for some $x \in S$, then (4.B) holds for every $x \in S$. Hence $A$ satisfies either (4.A) or (5.A), (4.A) implies (4.B), and (5.A) implies (5.B).

(IV) $S$ is closed (hence $e \in S$ by (i)), each of $\alpha, \beta, \gamma, \chi$ is continuous, and $\alpha$ is a semi-isomorphism onto a closed subsemigroup of $G^{\prime}$.

In fact, $S$ is closed by Lemma 4.3. To prove the continuity of $\alpha$ and $\gamma$, suppose $\left(x_{n}\right)$ is a convergent net in $S$ with limit $x$. Then $\left(\delta_{x_{n}}\right)$ is a tight net for $\delta_{x}$ in $M(S)$. Since $A$ is tightly continuous by Lemma 4.3 , it follows that $A\left(\delta_{x_{n}}\right) \rightarrow A\left(\delta_{x}\right)$ weak-*. In light of $(1)$, this means that $\gamma\left(x_{n}\right) \rightarrow \gamma(x)$ and $\alpha\left(x_{n}\right) \rightarrow \alpha(x)$. Hence both $\gamma$ and $\alpha$ are continuous, and so both $\chi$ and $\beta$ are also continuous by (II).

Now $\alpha$ is a semihomomorphism; hence $\alpha(S)$ is a subsemigroup of $G^{\prime}$. Moreover, $\alpha$ is an injection by (1) since $A$ is an additive injection. To prove that $\alpha(S)$ is closed in $G^{\prime}$, let $x^{\prime} \in G^{\prime}$ be a cluster point of $\alpha(S)$. Then there exists a net $\left(x_{n}\right)$ in $S$ such that $\alpha\left(x_{n}\right) \rightarrow x^{\prime}$. By (1), this means

$$
\overline{\gamma\left(x_{n}\right)} A\left(\delta_{x_{n}}\right)=\delta_{\alpha\left(x_{n}\right)} \rightarrow \delta_{x^{\prime}} \text { weak-*. }
$$

Since $A(M(S))$ is a weak-* closed subspace of $M\left(G^{\prime}\right)$, it follows that $\delta_{x^{\prime}} \in$ $A(M(S))$. It is now obvious from the proof of (I) that $x^{\prime}=\alpha(x)$ for some $x \in S$. Hence $\alpha(S)$ is closed in $G^{\prime}$.

(V) Suppose that $A$ satisfies (4.A). Then (1) ensures that $A$ is linear on $M_{d}(S)$ (the discrete measures) and so $A \mu=(\gamma \mu) \circ \alpha^{-1}$ for each $\mu \in M_{d}(S)$. Given $\mu \in M(S), M_{d}(S)$ has a tight net $\left(\mu_{n}\right)$ for $\mu$. Moreover, $A$ is tightly continuous by Lemma 4.3 and $\alpha: S \rightarrow G^{\prime}$ is continuous by (IV). It follows that

$$
A \mu=*-\lim A \mu_{n}=*-\lim \left(\gamma \mu_{n}\right) \circ \alpha^{-1}=(\gamma \mu) \circ \alpha^{-1} \text {. }
$$

Hence $A$ is linear and satisfies condition (a) in Theorem 4. It is easy to check that $A$ is a homomorphism (resp. an antihomomorphism) if and only if $\alpha$ is a homomorphism (resp. an antihomomorphism). Moreover, writing $c=\chi(e)$ and $z=\beta(e)$, we have

$$
\begin{aligned}
B\left(\delta_{x}\right) & =\chi(x) \delta_{\beta(x)} \quad \text { by }(2) \\
& =c \gamma(x) \delta_{z \alpha(x)} \quad \text { by (II) } \\
& =c \delta_{z} * \gamma(x) \delta_{\alpha(x)} \\
& =c \delta_{z} * A\left(\delta_{x}\right)=A\left(\delta_{x}\right) * c \delta_{z} \quad \text { by (1) and (II). }
\end{aligned}
$$


Since $A$ satisfies (4.A), $B$ must satisfy (4.B) by (III). Hence an argument similar to the above one shows that

$$
B \mu=c \delta_{z} * A \mu=(A \mu) * c \delta_{z} \forall \mu \in M(S) .
$$

If $A$ satisfies (5.A), then we can similarly show that $A$ is conjugately linear and satisfies (b), and (6) holds. This completes the proof of Theorem 4.

Corollary 5. Let $S, A, B, \alpha, \gamma$ be as in Theorem 4. Suppose that for each $x \in S$, there exists $\mu \in M(S)$ such that

$$
\limsup _{n}\left\|\delta_{\alpha\left(x_{n}\right)} * A \mu-\delta_{\alpha(x)} * A \mu\right\|<2\|\mu\|
$$

whenever $\left(x_{n}\right)$ is a net in $S$ such that $\alpha\left(x_{n}\right) \rightarrow \alpha(x)$. Then $\alpha$ is an (injective) homeomorphism.

Proof. We shall only confirm the result for the case that $\alpha$ is a homomorphism. Replacing $A$ by $\mu \rightarrow A(\bar{\gamma} \mu)$, we may suppose $\gamma=1$. Thus $x \in S$ and $\mu \in M(S)$ implies $A\left(\delta_{x} * \mu\right)=A \delta_{x} * A \mu=\delta_{\alpha(x)} * A \mu$.

Now suppose that the continuous bijection $\alpha: S \rightarrow \alpha(S)$ is not a homeomorphism. Then there exists $x \in S$ such that $\alpha^{-1}$ is not continuous at $\alpha(x)$. Since $S$ is a closed subset of the locally compact space $G$, it follows that for each neighborhood $V$ of $\alpha(x), \alpha^{-1}(V)$ has noncompact closure in $G$. Therefore $S$ has a net $\left(x_{n}\right)$ such that $\alpha\left(x_{n}\right) \rightarrow \alpha(x)$ but $\left(x_{n}\right)$ recedes to the point at infinity in the one-point compactification of $G$. Fix any $\mu \in M(S)$. Then $\delta_{x_{n}} * \mu$ and $\delta_{x} * \mu$ are eventually concentrated on "almost disjoint" sets; hence $\left\|\delta_{x_{n}} * \mu-\delta_{x} * \mu\right\| \rightarrow 2\|\mu\|$. Since $A$ is an additive isometry, it follows that

$$
\begin{aligned}
2\|\mu\| & =\lim _{n}\left\|A\left(\delta_{x_{n}} * \mu-\delta_{x} * \mu\right)\right\| \\
& =\lim _{n}\left\|\delta_{\alpha\left(x_{n}\right)} * A \mu-\delta_{\alpha(x)} * A \mu\right\| .
\end{aligned}
$$

Since $\mu \in M(S)$ was arbitrary, this contradicts our additional assumption, which completes the proof.

Corollary 6. Suppose, in addition to the hypotheses of Theorem 4, that there exists $\mu \in M(S)$ such that $A \mu$ has a nonzero absolutely continuous component. Then the mapping $\alpha$ in Theorem 4 is a homeomorphism.

Proof. If $\mu \in M(S)$ is as above, then it satisfies condition $(*)$ in Corollary 5 at each $x \in S$. Hence $\alpha$ is a homeomorphism.

Theorem 7. Suppose that $S \subset G$ is a subsemigroup with nonempty interior, and that $T: M(S) \rightarrow M\left(G^{\prime}\right)$ is an additive isometry such that $T(M(S))$ is a weak-* closed L-subspace of $M\left(G^{\prime}\right)$ and $T(\mu * \mu)=T \mu * T \mu$ for each $\mu \in M(S)$. Then $S$ is necessarily closed in $G$, and there exist a unique continuous homomorphism $\gamma: S \rightarrow \mathbb{T}$ and a unique continuous semi-isomorphism a from $S$ onto a closed subsemigroup of $G^{\prime}$ such that either

(a) $T \mu=(\gamma \mu) \circ \alpha^{-1} \forall \mu \in M(S)$, or

(b) $T \mu=(\gamma \bar{\mu}) \circ \alpha^{-1} \forall \mu \in M(S)$.

In particular, $T$ is a semi-homomorphism which is either linear or conjugately linear.

Proof. In the proof of Theorem 4, take $A=B=T$ and invoke Proposition 1 instead of Corollary 2. 
Corollary 8. Let $S \subset G$ be a closed subsemigroup with nonempty interior, let $T: M(S) \rightarrow M(S)$ be a linear surjective isometry which preserves convolution squares, and let $n \geq 2$ be a natural number. Then $T^{n}=I$ if and only if there exist a continuous homomorphism $\chi: S \rightarrow \mathbb{T}$ and a homeomorphic semiisomorphism $\alpha$ on $S$ such that (i) $T \mu=\chi \cdot\left(\mu \circ \alpha^{-1}\right)$ for $\mu \in M(S)$, (ii) $\alpha^{n}=$ id on $S$, and (iii) $\chi\left(\alpha(x) \alpha^{2}(x) \cdots \alpha^{n}(x)\right)=1$ for $x \in S$.

Proof. Let $\gamma$ and $\alpha$ be as in the conclusion of Theorem 7. Then $\alpha$ is a homeomorphism. (To see this, simply consider $T^{-1}$.) Since $T$ is linear, we must have $T \mu=(\gamma \mu) \circ \alpha^{-1}$. Set $\chi=\gamma \circ \alpha^{-1}$, so $\chi$ is a continuous character of $S$ and satisfies (i).

Now define $D: C_{0}(S) \rightarrow C_{0}(S)$ by setting $D f=(\chi f) \circ \alpha$. Then (i) shows that

$$
\int f d(T \mu)=\int \chi f d\left(\mu \circ \alpha^{-1}\right)=\int D f d \mu \forall f \in C_{0}(S) \text { and } \mu \in M(S) .
$$

In other words, $T$ is the adjoint mapping of $D$. Moreover,

$$
\left(D^{n} f\right)(x)=\chi\left(\alpha(x) \alpha^{2}(x) \cdots \alpha^{n}(x)\right) f\left(\alpha^{n} x\right)
$$

by induction. Therefore $T^{n}=I$ if and only if $\alpha$ and $\chi$ satisfy (ii) and (iii), which completes the proof.

We say that a measurable subset $E$ of $G$ is Haar-perfect if each relatively open nonempty subset of $E$ has positive Haar measure. An example of a Haar-perfect closed subsemigroup with nondense interior is obtained by taking $G=\mathbb{R}$ and $S=E \cup[2, \infty)$, where $E$ is any nonempty Lebesgue-perfect compact subset of $[1,2]$ with empty interior.

Theorem 9. Suppose (i) $S \subset G$ is a Haar-perfect closed subsemigroup containing $e$ and each of $S^{\prime}$ and $S^{\prime \prime}$ is a Haar-perfect closed subsemigroup of $G^{\prime}$, (ii) each of $A: M_{a}(S) \rightarrow M_{a}\left(S^{\prime}\right)$ and $B: M_{a}(S) \rightarrow M_{a}\left(S^{\prime \prime}\right)$ is an isometric additive surjection, and (iii) $A$ and $B$ satisfy

$$
B(\mu * \nu+\nu * \mu)=B \mu * A \nu+A \nu * B \mu \forall \mu, \nu \in M_{a}(S) .
$$

Then there exist a unique continuous homomorphism $\gamma: S \rightarrow \mathbb{T}$ and a unique homeomorphic quasi-isomorphism $\alpha: S \rightarrow S^{\prime}$ which satisfy either (a) or (b) of Theorem 4 with $M(S)$ replaced by $M_{a}(S)$. Moreover, there exist $c \in \mathbb{T}$ and $z \in S^{\prime \prime}$ such that $B \mu=\left(c \delta_{z}\right) * A \mu=A \mu *\left(c \delta_{z}\right)$ for each $\mu \in M_{a}(S)$.

Proof. (I) Arguing as in the proof of Lemma 4.3, one checks that $A$ extends uniquely to a tightly continuous mapping $A^{\prime}: M(S) \rightarrow M\left(S^{\prime}\right)$. (This requires Lemma 4.2 for $G^{\prime}$, but not for $G$.) Moreover, $A^{\prime}$ is a norm-decreasing additive mapping which satisfies $(\mathrm{J})$ with $A$ replaced by $A^{\prime}$ for $\mu \in M_{a}(S)$ and $\nu \in$ $M(S)$.

Similarly, $B$ extends uniquely to a tightly continuous mapping $B^{\prime}: M(S) \rightarrow$ $M\left(S^{\prime}\right)$, which is a norm-decreasing additive mapping satisfying

$$
B^{\prime}(\mu * \nu+\nu * \mu)=B^{\prime} \mu * A^{\prime} \nu+A^{\prime} \nu * B^{\prime} \mu \forall \mu, \nu \in M(S) .
$$

Similarly, $B^{-1}$ extends to a unique tightly continuous mapping $B^{\sim}: M\left(S^{\prime \prime}\right) \rightarrow$ $M(S)$, which is norm-decreasing and additive.

(II) We shall show, without using the assumption " $e \in S$ ", that each of $A^{\prime}$ and $B^{\prime}$ is an isometric surjection. 
To this end, choose and fix any $\mu_{0} \in M(S)$ such that $a \mu_{0} \geq 0$ for some nonzero $a \in \mathbb{C}$. Let $\nu \in M_{a}(S)$ be any probability measure; such a $\nu$ exists by (i). Since $M_{a}(S)$ is a two-sided ideal in $M(S)$ and each of $A$ and $B$ is an isometry, we infer from (1) that

$$
\begin{aligned}
2\left\|\mu_{0}\right\| & =\left\|\mu_{0} * \nu+\nu * \mu_{0}\right\|=\left\|B\left(\mu_{0} * \nu+\nu * \mu_{0}\right)\right\| \\
& \leq 2\left\|B^{\prime} \mu_{0}\right\| \cdot\|A \nu\|=2\left\|B^{\prime} \mu_{0}\right\|,
\end{aligned}
$$

and so $\left\|B^{\prime} \mu_{0}\right\|=\left\|\mu_{0}\right\|$.

Next choose any net $\left(\mu_{n}\right)$ in $M_{a}(S)$ such that $\left\|\mu_{n}\right\| \leq\left\|\mu_{0}\right\|$ for each $n$ and $\mu_{n} \rightarrow \mu_{0}$ weak-*. Such a net is necessarily a tight net [6]. Hence $B \mu_{n} \rightarrow B^{\prime} \mu_{0}$ weak-* by the tight continuity of $B^{\prime}$ (or by the definition of $B^{\prime}$ ) and $\left\|B \mu_{n}\right\|=$ $\left\|\mu_{n}\right\| \leq\left\|\mu_{0}\right\|=\left\|B^{\prime} \mu_{0}\right\|$ for each $n$. Therefore $\left(B \mu_{n}\right)$ is a tight net for $B^{\prime} \mu_{0}$ in $M_{a}\left(S^{\prime \prime}\right)$. It follows from the tight continuity of $B^{\sim}$ that

$$
B^{\sim}\left(B^{\prime} \mu_{0}\right)=*-\lim _{n} B^{\sim}\left(B \mu_{n}\right)=*-\lim _{n} \mu_{n}=\mu_{0} .
$$

Since $\mu_{0} \in M(S)$ was an arbitrary measure such that $a \mu_{0} \geq 0$ for some nonzero $a \in \mathbb{C}$ and since each of $B^{\sim}$ and $B^{\prime}$ is real-linear, we infer from (2) that $B^{\sim}\left(B^{\prime} \mu\right)=\mu$ for all $\mu \in M(S)$. Similarly, $B^{\prime}\left(B^{\sim} \mu^{\prime}\right)=\mu^{\prime}$ for all $\mu^{\prime} \in M\left(S^{\prime \prime}\right)$. Since each of $B^{\prime}$ and $B^{\sim}$ is norm-decreasing, we conclude that $B^{\prime}: M(S) \rightarrow$ $M\left(S^{\prime \prime}\right)$ is an isometric surjection. Similarly $A^{\prime}: M(S) \rightarrow M\left(S^{\prime}\right)$ is an isometric surjection.

(III) Accordingly, $S, A^{\prime}$, and $B^{\prime}$ fulfill all the hypotheses of Theorem 4. Hence there exist a unique continuous homomorphism $\gamma: S \rightarrow \mathbb{T}$ and a unique continuous quasi-isomorphism $\alpha: S \rightarrow S^{\prime}$ that satisfy either (a) or (b) of Theorem 4 with $A$ replaced by $A^{\prime}$. Moreover, $\alpha$ is a homeomorphism by Corollary 6. Since $A^{\prime}$ is an extension of $A$, this completes the proof.

Remark 10. (i) In light of Theorem 9 and its proof, both Theorem 7 and Corollary 8 hold with $M(S)$ replaced by $M_{a}(S)$ whenever $S$ is a nonempty Haarperfect closed subsemigroup. Corollary 8 and its "conjugately-linear" version are generalizations of the characterization of isometric involutions on $M(G)$ and on $M_{a}(G)$ by Patterson [7].

(ii) The author has been unable to remove the assumption " $e \in \bar{S}$ " in Theorem 4 and in Theorem 9. However, he conjectures that the isometry condition on $A$ (and on $B$ ) can be replaced by the weaker condition that $A$ is an injection with $\|A\|<(1+\sqrt{2}) / 2$. This conjecture is based upon the author's result [9] on idempotent measures and W. Rudin's observation in [8, 4.6.3(c)].

(iii) Let $G$ be an arbitrary locally compact group, and let $K$ be a compact infinite group. Define $A \mu=B \mu=\mu \times \lambda$ for $\mu \in M(G)$, where $\lambda$ is the norm-one Haar measure on $K$. Then $A$ is a linear isometric isomorphism of $M(G)$ onto a weak-* closed subalgebra of $M(G \times K)$. However, there exist no mappings $\gamma, \alpha$ as in the conclusion of Theorem 4 . Hence the " $L$-subspace" assumption in Theorem 4 is not superfluous.

(iv) Suppose $G$ is connected, $\mu \in M(G)$, and $D:=\left\{x \in G \mid \mu * \delta_{x}+\delta_{x} * \mu=0\right\}$ has nonempty interior. Then $\mu=0$. (This is obvious from the proof of Lemma 4.2.)

(v) Suppose the subsemigroup $S$ contains $e \in G$. Then the "squarepreserving" assumption on $T$ in Theorem 7 may be replaced by the "inversepreserving" assumption: $T\left(\mu^{-1}\right)=(T \mu)^{-1}$ whenever $\mu \in M(S)$ is invertible in $M(S)$. 
(vi) The Referee's Proof of Lemma 4.2: Let $T_{y}(\mu)=\delta_{y-1} * \mu * \delta_{y}$. Then $T_{y}(\mu)=-\mu$ for $y=x$ and $x^{2}$ by $(*)$. Hence

$$
\mu=T_{x}(-\mu)=T_{x} T_{x}(\mu)=-\mu,
$$

and so $\mu=0$, as desired.

\section{REFERENCES}

1. E. Artin, Geometric algebra, Interscience Tracts in Pure and Appl. Math., no. 3, Interscience, New York, 1957.

2. E. Hewitt and K. A. Ross, Abstract harmonic analysis, Vol. 1, Springer-Verlag, New York, Heidelberg, and Berlin, 1963.

3. N. Jacobson and C. E. Rickart, Jordan homomorphisms of rings, Trans. Amer. Math. Soc. 69 (1950), 479-502.

4. B. E. Johnson, Isometric isomorphisms of measure algebras, Proc. Amer. Math. Soc. 15 (1964), 186-187.

5. S. Kakutani, Concrete representation of abstract $(L)$-spaces and the mean ergodic theorem, Ann. of Math. (2) 42 (1941), 523-537.

6. M. Loève, Probability theory. I, 4th ed., Springer-Verlag, New York, Heidelberg, and Berlin, 1977.

7. P. L. Patterson, Involutions on algebras arising from locally compact groups, preprint, 1992.

8. W. Rudin, Fourier analysis on groups, Interscience Tracts in Pure and Appl. Math., no. 12, Interscience, New York, 1962.

9. S. Saeki, On norms of idempotent measures, Proc. Amer. Math. Soc. 19 (1968), 600-602.

10. W. R. Scott, Half-homomorphisms of groups, Proc. Amer. Math. Soc. 8 (1957), 1141-1144.

11. L. N. Ševrin, On semi-isomorphisms and lattice isomorphisms of semigroups with cancellation, Soviet Math. Dokl. 7 (1966), 1491-1493.

12. _ Half-isomorphisms of cancellative semigroups, Math. USSR-Izv. 1 (1967), 915-921.

13. J. L. Taylor, Measure algebras, CBMS Regional Conf. Ser. in Math., no. 16, Amer. Math. Soc., Providence, RI, 1972.

14. J. G. Wendel, On isometric isomorphism of group algebras, Pacific J. Math. 1 (1951), 305-312.

Department of Mathematics, Kansas State University, Manhattan, Kansas 66502

E-mail address: math@ksuvm.ksu.edu 